\title{
LED-UV-Systeme für industrielle Anwendungen
}

st Metz nutzte die JEC Europe (11. - 13. März, Paris), um den Messebesuchern UV-Systeme für klebtechnische Anwendungen in den Bereichen Elektronik, Optoelektronik und Medizintechnik sowie für die Verklebung von Glas, Kunststoffen und Metall vorzustellen.

Zum Angebot des Unternehmens zählen sowohl konventionelle UV-Systeme als auch LED-Aggregate zur Aushärtung von kationischen, radikalischen oder Dual-Cure-Klebstoffen.

Die UV-Systeme sind bezüglich Wellenlänge und Intensität des UV-Lichts auf die speziellen Klebstoffanforderungen abgestimmt und härten die Klebstoffe in Sekundenschnelle aus, sodass das Material sofort weiterverarbeitet werden kann. Mit der Aushärtung durch UV-Licht wird eine hohe Festigkeit und Beständigkeit erreicht. Dies gilt auch für die Fertigung und Verklebung von Verbundmaterialien.

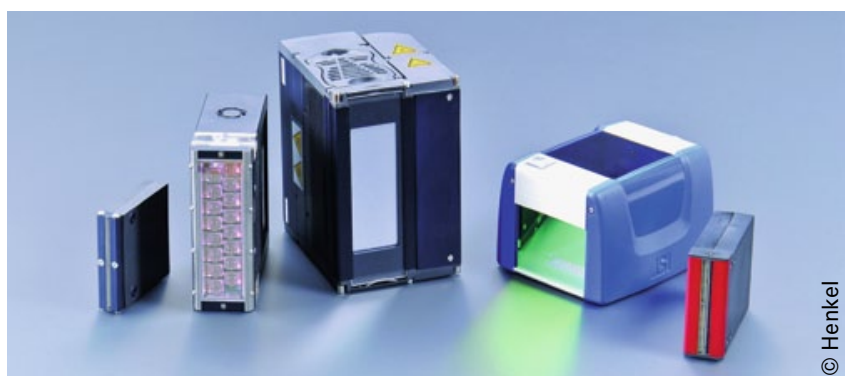

Ist Metz präsentierte auf der JEC Europe UV-Systeme, die in den Bereichen Elektronik, Optoelektronik und Medizintechnik sowie für die Verklebung von Glas, Kunststoffen und Metall zum Einsatz kommen.

Weitere Infos: Ist Metz GmbH, Nürtingen, www.ist-uv.com

\section{Schlauch für alle Fälle Autarke Dosiereinheit}

$D^{i}$ Schläuche der Master-PUR-Serie wurden modifiziert und bieten jetzt sowohl Mikrobenresistenz als auch antistatische Eigenschaften. Durch die Verbindung dieser beiden Eigenschaften lassen sich die Lagerhaltung optimieren, Reaktions- und Lieferzeiten verkürzen und Kosten einsparen.

Die Schlauchreihe besteht aus reinem Polyester-Polyurethan mit einer dreh- sowie verschiebefest eingegossenen Federstahl-Spirale und wird mit unterschiedlichen Wandstärken von leicht über mittelschwer bis hin zu verstärkten Ausführungen angeboten.

Weitere Infos: Masterflex SE, www.masterflex.de

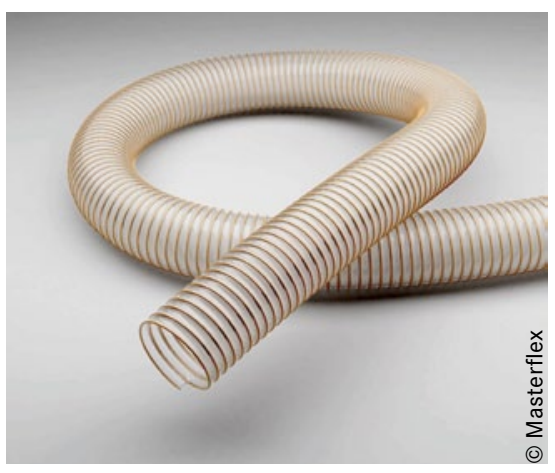

Zum Absaugen oder Fördern stehen modifiziert Schäuche zur Verfügung.
O erlikon Barmag hat eine kompakte Dosiereinheit auf den Markt gebracht, die direkt steuerbar ist, aber auch in vorhandene Prozesssteuerungen nachträglich integriert werden kann.

Ob beim Vergießen von PUR-Formteilen, beim Laminieren von Composite-Bauteilen, bei der Zugabe von Additiven in einen laufenden Extrusionsprozess, beim Auftragen von Kaltleim oder beim flexiblen Einsatz in Produktionsanlagen mit wechselnden Anforderungen die neue mobile Einheit ist in der Lage, diese Aufgaben mit hoher Dosiergenauigkeit auf einfache Weise zu unterstützen.

Die bedienerfreundliche Steuerung über Touchscreen erlaubt die Vorgabe von Dosiermengen, auch variabel in Abhängigkeit vom Hauptproduktionsprozess. Ebenso können alle anderen erforderlichen Parameter einfach vorgegeben werden. Alle wesentlichen Prozesswerte werden auf dem Touchscreen übersichtlich angezeigt. Weiterhin sind Druck- und Tankfüllstandsüberwachung vorgesehen. Die Kommunikation mit weiteren Dosieroder Mischsystemen erfolgt über CANBus-Schnittstellen oder über einen Netzwerk-(LAN-)Anschlgestaltuss. Schnellkupplungen an Ein- und Austritt erlauben

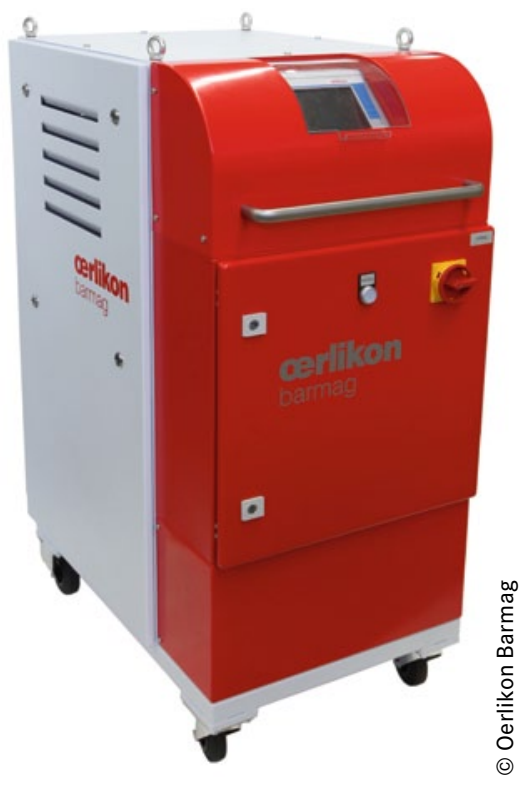

Diese mobile Dosiereinheit ist direkt ansteuerbar, lässt sich bei Bedarf aber auch in vorhandene Prozesssteuerungen nachträglich integrieren.

eine leckagefreie Verbindung zum Versorgungstank und zur Produktleitung.

Je nach gewünschter Durchsatzmenge kann aus einer Vielzahl von Förderkapazitäten gewählt werden.

Weitere Infos: Oerlikon Textile GmbH \& Co. KG, Remscheid, www.oerlikontextile.com 